JOURNAL OF

FUNCTION SPACES AND APPLICATIONS

Volume 5, Number 3 (2007), 287-297
(C) 2007, Scientific Horizon http://www.jfsa.net

\title{
Bloch-type space of temperature functions on a finite cylinder
}

\section{Marcos López-García}

(Communicated by Hans Triebel)

2000 Mathematics Subject Classification. Primary: 46E22, 47B38; Secondary: 35K05.

Keywords and phrases. Bloch space, duality, temperature functions.

Abstract. We define the Bloch-type space $\mathcal{B}_{T}$ as the linear space of temperature functions on the cylinder $S_{T}=\mathbb{S}^{1} \times(0, T)$ such that $\sup _{(x, t) \in \Omega_{T}} t\left|\frac{\partial u}{\partial t}(x, t)\right|<\infty, \Omega_{T}=[0,2] \times(0, T) ;$ we prove that $\left(b^{1}\left(S_{T}\right)\right)^{*}=\mathcal{B}_{T}$, $(x, t) \in \Omega_{T}$

where $b^{1}\left(S_{T}\right)$ is the Bergman space of temperature functions on $S_{T}$ belonging to $L^{1}\left(\Omega_{T}, d x d t\right)$.

\section{Introduction}

For $\mathbb{D}$ the open unit disk in the complex plane $\mathbb{C}$, the classic Bergman space $A^{p}$ is the subspace of analytic functions $f: \mathbb{D} \rightarrow \mathbb{C}$ such that $f \in L^{p}(\mathbb{D}), 0<p<\infty$. If $1 \leq p<\infty$ then $A^{p}$ is a Banach space, and for $p>1$ it is well known that $\left(A^{p}\right)^{*}=A^{q}$ under the integral pairing (see [4, Theorem 1.16])

$$
\langle f, g\rangle=\int_{\mathbb{D}} f(z) \overline{g(z)} d A(z), \quad f \in A^{p}, g \in A^{q},
$$


where $d A(z)=\pi^{-1} d x d y$, and $q$ is the conjugate exponent of $p: p^{-1}+q^{-1}=$ 1.

An analytic function $f$ in $\mathbb{D}$ is in the Bloch space $\mathcal{B}$ if $\sup _{z \in \mathbb{D}}(1-$ $\left.|z|^{2}\right)\left|f^{\prime}(z)\right|<\infty$. When normed with

$$
\|f\|_{\mathcal{B}}=|f(0)|+\sup _{z \in \mathbb{D}}\left(1-|z|^{2}\right)\left|f^{\prime}(z)\right|,
$$

the Bloch space $\mathcal{B}$ is a Banach space. In addition, we have $\left(A^{1}\right)^{*}=\mathcal{B}$ under the integral pairing (see [4, Theorem 1.21])

$$
\langle f, g\rangle=\lim _{r \rightarrow 1^{-}} \int_{\mathbb{D}} f(r z) \overline{g(z)} d A(z), \quad f \in A^{1}, g \in \mathcal{B} .
$$

For $\Omega \subset \mathbb{R}_{+}^{2}$ an open set, let

$$
H(\Omega)=\left\{u \in C^{2}(\Omega): \frac{\partial^{2} u}{\partial x^{2}}=\frac{\partial u}{\partial t} \text { on } \Omega\right\} .
$$

We shall call the elements of $H(\Omega)$ temperature functions on $\Omega$.

For $T>0$ finite, we define the linear space of temperature functions on the cylinder $S_{T}=\mathbb{S}^{1} \times(0, T)$ as

$$
H\left(S_{T}\right)=\{u \in H(\mathbb{R} \times(0, T)): u(x, t)=u(x+2, t)\} .
$$

Roughly speaking, a function in $H\left(S_{T}\right)$ determines a temperature distribution on a circular ring of fine wire with radius $1 / \pi$, up to a certain time $\mathrm{T}$.

In [7] we introduced the weighted Bergman-type spaces $b_{\alpha}^{p}\left(S_{T}\right)$ consisting of temperature functions on the cylinder $S_{T}$ that belong to $L_{\alpha}^{p}\left(\Omega_{T}\right)=$ $L^{p}\left(\Omega_{T}, t^{\alpha} d x d t\right)$, where $\Omega_{T}=[0,2] \times(0, T), 1 \leq p<\infty$, and $\alpha>-1$. Notice that if $u \in b_{\alpha}^{p}\left(S_{T}\right)$ then $\left.u\right|_{\Omega_{T}} \in L_{\alpha}^{p}\left(\Omega_{T}\right)$, hence $b_{\alpha}^{p}\left(S_{T}\right) \subset L_{\alpha}^{p}\left(\Omega_{T}\right)$.

As in the analytic case, we proved that $\left(b_{\alpha}^{p}\left(S_{T}\right),\|\cdot\|_{L^{p}\left(\Omega_{T}\right)}\right)$ is a Banach space, and $\left(b_{\alpha}^{p}\left(S_{T}\right)\right)^{*}=b_{\left(\beta-\frac{\alpha}{p}\right) q}^{q}\left(S_{T}\right)$ under the integral pairing (see [7, Theorem 1.2])

$$
\langle u, v\rangle_{\beta}=\int_{\Omega_{T}} u(z) \overline{v(z)} t^{\beta} d x d t, \quad z=(x, t),
$$

provided that $\alpha, \beta>-1, p>\max \left(1, \frac{1+\alpha}{1+\beta}\right)$. In particular, for $\alpha>-1$ we have that $b_{\alpha}^{2}\left(S_{T}\right)$ is a Hilbert space with respect the inner product inherited from $L_{\alpha}^{2}\left(\Omega_{T}\right)$,

$$
(u, v)_{b_{\alpha}^{2}\left(S_{T}\right)}=\int_{\Omega_{T}} u(z) \overline{v(z)} t^{\alpha} d x d t,
$$

and the corresponding orthogonal projection $P_{\alpha}: L_{\alpha}^{2}\left(\Omega_{T}\right) \rightarrow b_{\alpha}^{2}\left(S_{T}\right)$ is called the weighted Bergman projection on $b_{\alpha}^{2}\left(S_{T}\right)$. 
In this paper we define the Bloch-type space $\mathcal{B}_{T}$ as the linear space of temperature functions $v$ on $S_{T}$ such that $\sup \left\{t\left|\frac{\partial v}{\partial t}(x, t)\right|:(x, t) \in \Omega_{T}\right\}<$ $\infty$. When $\mathcal{B}_{T}$ is endowed with the norm

$$
\|v\|_{\mathcal{B}_{T}}=\max _{x \in[0,2]}\left|v\left(x, \frac{T}{2}\right)\right|+\sup \left\{t\left|\frac{\partial v}{\partial t}(x, t)\right|:(x, t) \in \Omega_{T}\right\},
$$

we obtain that $\left(\mathcal{B}_{T},\|\cdot\|_{\mathcal{B}_{T}}\right)$ is a Banach space. Furthermore, we show that $\left(b^{1}\left(S_{T}\right)\right)^{*}=\mathcal{B}_{T}$ under the integral pairing

$$
\langle u, v\rangle_{0}=\int_{\Omega_{T}} u(z) \overline{\left(\mathcal{L}_{0}^{1} v\right)(z)} d x d t, \quad u \in b^{1}\left(S_{T}\right), v \in \mathcal{B}_{T},
$$

where $\mathcal{L}_{0}^{1}: \mathcal{B}_{T} \rightarrow L^{\infty}\left(\Omega_{T}\right)$ is a bounded operator such that $P_{0} \mathcal{L}_{0}^{1} v=v$ for every $v \in \mathcal{B}_{T}$.

In fact, we have the following results,

Theorem 1. For $\alpha>-1$, there is a bounded linear differential operator $\mathcal{L}_{\alpha}^{1}: \mathcal{B}_{T} \rightarrow L^{\infty}\left(\Omega_{T}\right)$ such that $P_{\alpha} \mathcal{L}_{\alpha}^{1} v=v$ for all $v \in \mathcal{B}_{T}$.

Theorem 2. If $\alpha>-1$ then $\left(b_{\alpha}^{1}\left(S_{T}\right)\right)^{*}=\mathcal{B}_{T}$ under the integral pairing

$$
\langle u, v\rangle_{\alpha}=\int_{\Omega_{T}} u(z) \overline{\left(\mathcal{L}_{\alpha}^{1} v\right)(z)} t^{\alpha} d x d t, \quad u \in b_{\alpha}^{1}\left(S_{T}\right), v \in \mathcal{B}_{T} .
$$

The proof of this theorem is based on the methods and techniques in [1], [3], [5], [6], which involve the construction of the so-called Bell operator $\mathcal{L}^{1}$ satisfying $P \mathcal{L}^{1} v=v$ for every $v \in \mathcal{B}$, where $P$ is the Bergman projection and $\mathcal{B}$ the Bloch space in the corresponding setting (harmonic functions on the unit ball in $\mathbb{R}^{n}$, holomorphic functions on strictly pseudoconvex domains with $C^{4}$ boundary in $\mathbb{C}^{n}$ ).

\section{Notation and preliminary results}

Throughout this paper we will write $z=(x, t), w=(y, \tau), d z=d x d t$, $d w=d y d \tau, \mathbb{S}^{1}=\left\{e^{\pi i \theta}: \theta \in[0,2]\right\}, \mathbb{Z}^{*}=\{n \in \mathbb{Z}: n \neq 0\}$, and $\alpha$ will be a real number greater than -1 . Also $K(x, t)$ will denote the GaussWeierstrass kernel. For $t>0$, let

$$
\begin{aligned}
\theta(x, t) & =\sum_{n \in \mathbb{Z}} K(x+2 n, t) \\
& =\frac{1}{2} \sum_{n \in \mathbb{Z}} e^{-\pi^{2} n^{2} t+\pi n i x}, \quad[9, \text { Chapter V, Theorem 4.1] } \\
\varphi(x, t) & =-2 \frac{\partial \theta}{\partial x}(x, t)
\end{aligned}
$$


and for $t \leq 0$, let $K=\theta=\varphi=0$. It follows that

$$
\int_{0}^{2} \theta(x, t) d x=\int_{-\infty}^{\infty} K(x, t) d x=1, \quad \text { for all } t>0 .
$$

Let $Q=(0,1) \times(0,1), \Gamma=\Gamma_{1} \cup \Gamma_{2} \cup \Gamma_{3}$ (the parabolic boundary of $Q$ ), where $\Gamma_{1}=\{0\} \times[0,1), \Gamma_{2}=\{1\} \times[0,1), \Gamma_{3}=(0,1) \times\{0\}$, and let $\lambda$ be the one-dimensional Lebesgue measure on $\Gamma$.

Consider the heat kernel $\widetilde{K}(x, t ; \xi, \tau)$ on $Q \times \Gamma$ defined as follows

$$
\widetilde{K}(x, t ; \xi, \tau)= \begin{cases}\varphi(x, t-\tau), & \xi=0,0 \leq \tau<1, \\ \varphi(1-x, t-\tau), & \xi=1,0 \leq \tau<1, \\ \theta(x-\xi, t)-\theta(x+\xi, t), & \tau=0,0<\xi<1 .\end{cases}
$$

It is well known that if $u \in H(Q) \cap C(\bar{Q})$ then (see [2, Theorem 6.3.1])

$$
u(x, t)=\int_{\Gamma} \widetilde{K}(x, t ; \xi, \tau) u(\xi, \tau) d \lambda(\xi, \tau), \text { for all }(x, t) \in Q .
$$

Conversely, if $v \in C(\Gamma)$ then

$$
u(x, t)=\int_{\Gamma} \widetilde{K}(x, t ; \xi, \tau) v(\xi, \tau) d \lambda(\xi, \tau)
$$

is a temperature function on $Q$.

Remark 3. Suppose $f$ is a 2 -periodic continuous function on $\mathbb{R}$. Then $u$ is a temperature function on $\mathbb{R}_{+}^{2}, 2$-periodic in the variable $x$, and $u(x, t) \rightarrow f(x)$ as $t \rightarrow 0$ uniformly on [0,2] if and only if (see [9, Chapter $\mathrm{V}$, Theorem 8])

$$
u(x, t)=\int_{0}^{2} \theta(x-y, t) f(y) d y .
$$

Next we give a basic but useful result.

Proposition 4. If $u \in H\left(S_{T}\right)$ then $|u(x, t)| \leq \max _{x \in[0,2]}\left|u\left(x, \frac{T}{2}\right)\right|$ for all $\frac{T}{2}<t<T$.

Proof. Let $u \in H\left(S_{T}\right)$, by the remark above and the uniqueness of the solution of the heat equation on a finite cylinder (see [9, p. 100]), we can write

$$
u(x, t)=\int_{0}^{2} \theta\left(x-y, t-\frac{T}{2}\right) u\left(y, \frac{T}{2}\right) d y, \text { for all } \frac{T}{2}<t<T,
$$

therefore

$$
\begin{aligned}
|u(x, t)| & \leq \max _{x \in[0,2]}\left|u\left(x, \frac{T}{2}\right)\right| \int_{-\infty}^{\infty} K\left(x-y, t-\frac{T}{2}\right) d y \\
& =\max _{x \in[0,2]}\left|u\left(x, \frac{T}{2}\right)\right|, \quad \text { for all } t>\frac{T}{2} .
\end{aligned}
$$


We define the linear space of continuous functions on the cylinder $\overline{S_{T}}$ as

$$
C\left(\overline{S_{T}}\right)=\{u \in C(\mathbb{R} \times[0, T]): u(x, t)=u(x+2, t)\} .
$$

For $1 \leq p<\infty, \alpha>-1$ the subspace $H\left(S_{T}\right) \cap C\left(\overline{S_{T}}\right)$ is dense in $b_{\alpha}^{p}\left(S_{T}\right)$ ([7, Corollary 3.8]).

We define the incomplete gamma function $\gamma$ as

$$
\gamma(\beta, \sigma)=\int_{0}^{\sigma} t^{\beta-1} e^{-t} d t \quad \text { if } \beta, \sigma>0 .
$$

For any $n \in \mathbb{Z}^{*}$, let

$$
u_{n}(x, t)=\sqrt{\frac{2^{\alpha} \pi^{2(1+\alpha)} n^{2(1+\alpha)}}{\gamma\left(1+\alpha, 2 \pi^{2} n^{2} T\right)}} e^{-\pi^{2} n^{2} t+\pi n i x},
$$

and $u_{0}(x, t)=\sqrt{\frac{1+\alpha}{2 T^{1+\alpha}}}$, then $\left\{u_{n}\right\}_{n \in \mathbb{Z}}$ is an orthonormal basis of $b_{\alpha}^{2}\left(S_{T}\right)$ ([7, Lemma 3.9]).

The weighted Bergman projection $P_{\alpha}: L_{\alpha}^{2}\left(\Omega_{T}\right) \rightarrow b_{\alpha}^{2}\left(S_{T}\right)$ can be written as the integral operator

$$
P_{\alpha} u(z)=\int_{\Omega_{T}} N_{\alpha}(z, w) u(w) \tau^{\alpha} d w,
$$

where $N_{\alpha}(z, w)$ is a symmetric, real-valued function called the Bergman reproducing kernel of $b_{\alpha}^{2}\left(S_{T}\right)$, it is given by

$$
N_{\alpha}(z, w)=\sum_{n \in \mathbb{Z}} u_{n}(z) \overline{u_{n}(w)}=\sum_{n \in \mathbb{Z}} u_{n}(x, t) \overline{u_{n}(y, \tau)},
$$

and satisfies $N_{\alpha}(\cdot, w) \in b_{\alpha}^{2}\left(S_{T}\right)$ for every $w \in S_{T}$ (see [7, Section 3]).

Since $\gamma\left(1+\alpha, 2 \pi^{2} n^{2} T\right) \geq \gamma\left(1+\alpha, 2 \pi^{2} T\right)$, and $e^{-x} \leq C_{\lambda} x^{-\lambda}$ for $x>0, \lambda \geq 0$ we have that the series defining $N_{\alpha}$ converges absolutely and uniformly on $\Omega^{\prime} \times \mathbb{R}_{+}^{2}$ provided $\Omega^{\prime} \subset \mathbb{R}_{+}^{2}$ is compact, furthermore, the function $N_{\alpha}$ and its derivatives are bounded on $\Omega^{\prime} \times \mathbb{R}_{+}^{2}$, therefore $N_{\alpha} \in C^{\infty}\left(\mathbb{R}_{+}^{2} \times \mathbb{R}_{+}^{2}\right)$ and $N_{\alpha}(\cdot, w) \in H\left(S_{T}\right)$ for all $w \in \mathbb{R}_{+}^{2}$. On the other hand, the function

$$
\Theta_{\alpha}(z, w)=\theta_{\alpha}(x-y, t+\tau)=\frac{1}{2} \pi^{2(1+\alpha)} \sum_{n \in \mathbb{Z}} n^{2(1+\alpha)} e^{-\pi^{2} n^{2}(t+\tau)+\pi n i(x-y)},
$$

satisfies ([8, Lema 2.14])

$$
\int_{\Omega_{T}}\left|\Theta_{\alpha}(z, w)\right| \tau^{\beta} d w \leq C_{\alpha, \beta} t^{\beta-\alpha} \text { if } \alpha>\beta>-1 .
$$

Finally, during the proof of Theorem (1.3) in [8] we have constructed an isomorphism $\mathcal{M}_{\alpha}: b_{\beta}^{1}\left(S_{T}\right) \rightarrow b_{\beta}^{1}\left(S_{T}\right)$, applying the theory of Fourier 
multipliers on $L^{1}\left(S^{1}\right)$, such that

$$
\begin{aligned}
& \mathcal{M}_{\alpha}\left(\frac{\partial^{n} N_{\alpha}}{\partial x^{n}}(z, w)\right)=i^{n} \Theta_{\alpha+\frac{n}{2}}(z, w), \text { if } \alpha+\frac{n}{2}>\beta>-1 \\
& \mathcal{M}_{\alpha}\left(\frac{\partial^{n} N_{\alpha}}{\partial t^{n}}(z, w)\right)=(-1)^{n} \Theta_{\alpha+n}(z, w), \text { if } \alpha+n>\beta>-1
\end{aligned}
$$

where $\mathcal{M}_{\alpha}$ is acting on the variable $w$. In particular, we have

$$
\text { (3) } \begin{aligned}
\int_{\Omega_{T}}\left|\frac{\partial N_{\alpha}}{\partial x}(z, w)\right| \tau^{\alpha} d w & \leq C_{\alpha} \int_{\Omega_{T}}\left|\Theta_{\alpha+\frac{1}{2}}(z, w)\right| \tau^{\alpha} d w \leq C_{\alpha} t^{-\frac{1}{2}}, \\
\int_{\Omega_{T}}\left|\frac{\partial N_{\alpha}}{\partial t}(z, w)\right| \tau^{\alpha} d w & \leq C_{\alpha} \int_{\Omega_{T}}\left|\Theta_{\alpha+1}(z, w)\right| \tau^{\alpha} d w \leq C_{\alpha} t^{-1} .
\end{aligned}
$$

\section{Basic properties of the Bloch-type space $\mathcal{B}_{T}$}

We recall the definition of the normed linear space $\mathcal{B}_{T}$.

Definition 5. For $T>0$, let

and

$$
\mathcal{B}_{T}=\left\{v \in H\left(S_{T}\right): \sup \left\{t\left|\frac{\partial v}{\partial t}(x, t)\right|:(x, t) \in \Omega_{T}\right\}<\infty\right\},
$$

$$
\|v\|_{\mathcal{B}_{T}}=\max _{x \in[0,2]}\left|v\left(x, \frac{T}{2}\right)\right|+\sup \left\{t\left|\frac{\partial v}{\partial t}(x, t)\right|:(x, t) \in \Omega_{T}\right\} .
$$

The following result is about the growth of functions in $\mathcal{B}_{T}$.

Lemma 6. If $v \in \mathcal{B}_{T}$ then

$$
|v(x, t)| \leq\|v\|_{\mathcal{B}_{T}}\left(1+\ln \frac{T}{t}\right), \text { for all }(x, t) \in \Omega_{T} .
$$

Proof. For $t<\frac{T}{2}$ we have

$$
\begin{aligned}
\left|v\left(x, \frac{T}{2}\right)-v(x, t)\right|= & \left|\int_{0}^{1} \frac{d}{d \sigma} v\left(x, \sigma \frac{T}{2}+(1-\sigma) t\right) d \sigma\right| \\
= & \left|\int_{0}^{1}\left(\frac{T}{2}-t\right) \frac{\partial v}{\partial t}\left(x, \sigma \frac{T}{2}+(1-\sigma) t\right) d \sigma\right| \\
\leq & \sup \left\{t\left|\frac{\partial v}{\partial t}(x, t)\right|:(x, t) \in \Omega_{T}\right\} \\
& \times \int_{0}^{1} \frac{\left(\frac{T}{2}-t\right)}{\sigma \frac{T}{2}+(1-\sigma) t} d \sigma \\
\leq & \|v\|_{\mathcal{B}_{T}} \ln \frac{T}{2 t} .
\end{aligned}
$$

When $t>\frac{T}{2}$ the inequality holds by Proposition 4 . 
Remark 7. From the 2-periodicity in the variable $x$ of the functions in $\mathcal{B}_{T}$, together with (4), we note that norm convergence in $\mathcal{B}_{T}$ implies the uniform convergence on compact subsets in $\mathbb{R} \times(0, T)$.

Next we show that the normed linear space $\left(\mathcal{B}_{T},\|\cdot\|_{\mathcal{B}_{T}}\right)$ is complete.

Proposition 8. For every $T>0, \mathcal{B}_{T}$ is a Banach space.

Proof. Let $\left(u_{j}\right)$ a Cauchy sequence in $\mathcal{B}_{T}$, by the remark above there is a function $u$ such that $u=\lim u_{j}$. Likewise, the sequence $\left(\frac{\partial}{\partial t} u_{j}\right)$ converges uniformly on compact subsets in $\mathbb{R} \times(0, T)$, therefore $\frac{\partial u_{j}}{\partial t} \rightarrow \frac{\partial u}{\partial t}$ on $\mathbb{R} \times(0, T)$.

Pick $\left(x_{0}, t_{0}\right) \in \Omega_{T}$. Let $R=(a, b) \times(c, d)$, with $d-c=(b-a)^{2}$, such that $\left(x_{0}, t_{0}\right) \in R$ and $\bar{R} \subset \Omega_{T}$. Consider the mapping $\Psi: Q \rightarrow R$ given by $\Psi(\xi, \tau)=((b-a) \xi+a,(d-c) \tau+c)$. Since $u_{j} \in H(R) \cap C(\bar{R}),(1)$ implies that

$u_{j}(x, t)=\int_{\Gamma_{R}} \widetilde{K}\left(\Psi^{-1}(x, t) ; \Psi^{-1}(\xi, \tau)\right) u_{j}(\xi, \tau) d \lambda_{R}(\xi, \tau), \quad$ for $(x, t) \in R$,

where $\Gamma_{R}=\Psi(\Gamma)$ and $\lambda_{R}$ is the one-dimensional Lebesgue measure normalized on each segment of $\Gamma_{R}$. By the dominated convergence theorem we have

$$
u(x, t)=\int_{\Gamma_{R}} \widetilde{K}\left(\Psi^{-1}(x, t) ; \Psi^{-1}(\xi, \tau)\right) u(\xi, \tau) d \lambda_{R}(\xi, \tau) .
$$

Since the function $u$ is continuous on $\Gamma_{R}$ then $u$ is a temperature function on $R$. Since $\left(x_{0}, t_{0}\right) \in \Omega_{T}$ was arbitrary we conclude that $u \in H\left(S_{T}\right)$, and $\left\|u-u_{j}\right\|_{\mathcal{B}_{T}} \rightarrow 0$ as $j \rightarrow \infty$.

Proposition 9. Let $1 \leq p<\infty$ and $\alpha>-1$. If $v \in \mathcal{B}_{T}$ then $v \in b_{\alpha}^{p}\left(S_{T}\right)$. Therefore $P_{\alpha} v=v$ for all $v \in \mathcal{B}_{T}$.

Proof. Since $t^{\alpha} d x d t$ is a finite measure on $\Omega_{T}$, by the previous lemma it is sufficient to show that $\int_{0}^{T}\left|\ln \frac{T}{t}\right|^{p} t^{\alpha} d t<\infty$. Let $\varepsilon>0$ such that $\varepsilon<\alpha+1$, then $|\ln u|^{p} / u^{\alpha+1-\varepsilon} \rightarrow 0$ as $u \rightarrow \infty$, so

$$
\int_{0}^{T}\left|\ln \frac{T}{t}\right|^{p} t^{\alpha} d t=T^{\alpha+1} \int_{1}^{\infty} \frac{|\ln u|^{p}}{u^{\alpha+2}} d u \leq C_{\alpha, T} \int_{1}^{\infty} \frac{1}{u^{1+\varepsilon}} d u<\infty .
$$

Proposition 10. For $\alpha>-1$ the weighted Bergman projection $P_{\alpha}$ maps $L^{\infty}\left(\Omega_{T}\right)$ continuously into $\mathcal{B}_{T}$. Additionally, $\left\|t^{1 / 2} \frac{\partial}{\partial x}\left(P_{\alpha} \varphi\right)\right\|_{L^{\infty}\left(\Omega_{T}\right)}<\infty$ for every $\varphi \in L^{\infty}\left(\Omega_{T}\right)$.

Proof. Let $\varphi \in L^{\infty}\left(\Omega_{T}\right)$, differentiating under the integral sign yields

$$
\frac{\partial\left(P_{\alpha} \varphi\right)}{\partial t}(z)=\int_{\Omega_{T}} \frac{\partial N_{\alpha}}{\partial t}(z, w) \varphi(w) \tau^{\alpha} d w, \quad z \in \Omega_{T}
$$


by (3) we get

$$
\left|\frac{\partial\left(P_{\alpha} \varphi\right)}{\partial t}(z)\right| \leq C_{\alpha}\|\varphi\|_{L^{\infty}\left(\Omega_{T}\right)} t^{-1}, \quad z \in \Omega_{T} .
$$

The proof of the other conclusion is similar.

Finally, we prove the main results.

Proof of Theorem 1. Let $\psi_{\alpha} \in C^{\infty}(\mathbb{R})$ a nonnegative function satisfying that $\psi_{\alpha} \equiv 1 /(\alpha+1)$ for $t \leq \frac{T}{2}$ and $\psi_{\alpha} \equiv 0$ for $t>T-\delta$, with $\delta>0$ small enough. Thus, for $v \in \mathcal{B}_{T}$ we define

$$
\begin{aligned}
\left(\mathcal{L}_{\alpha}^{1} v\right)(x, t) & =v(x, t)-\left[\alpha \psi_{\alpha}(t) v(x, t)+\left(\frac{\partial}{\partial t}+\frac{\partial^{2}}{\partial x^{2}}\right)\left(\psi_{\alpha}(t) t v(x, t)\right)\right] \\
& =\left\{1-(1+\alpha) \psi_{\alpha}(t)-t \psi_{\alpha}^{\prime}(t)\right\} v(x, t)-2 t \psi_{\alpha}(t) \frac{\partial v}{\partial t}(x, t) .
\end{aligned}
$$

Clearly $\mathcal{L}_{\alpha}^{1}$ is a linear differential operator on $\mathcal{B}_{T}$. By Proposition 4 , it follows that

$$
\left|\left(\mathcal{L}_{\alpha}^{1} v\right)(x, t)\right| \leq C_{\psi_{\alpha}}\|v\|_{\mathcal{B}_{T}} \text { for all }(x, t) \in \Omega_{T} .
$$

Letting $g(x, t)=\alpha \psi_{\alpha}(t) v(x, t)+\left(\frac{\partial}{\partial t}+\frac{\partial^{2}}{\partial x^{2}}\right)\left(\psi_{\alpha}(t) t v(x, t)\right)$, we claim that $g \in L_{\alpha}^{2}\left(\Omega_{T}\right)$, and $(g, u)_{L_{\alpha}^{2}\left(\Omega_{T}\right)}=0$ for all $u \in H\left(S_{T}\right) \cap C\left(\overline{S_{T}}\right)$. So, $P_{\alpha} \mathcal{L}_{\alpha}^{1} v=P_{\alpha} v-P_{\alpha} g=P_{\alpha} v=v$ since $v \in b_{\alpha}^{2}\left(S_{T}\right)$.

To prove the claim, we rewrite $g=\left((1+\alpha) \psi_{\alpha}+t \psi_{\alpha}^{\prime}\right) v+2 t \psi_{\alpha} \frac{\partial v}{\partial t}$ then $g \in L_{\alpha}^{2}\left(\Omega_{T}\right)$. By the other hand, if $u \in H\left(S_{T}\right) \cap C\left(\overline{S_{T}}\right)$ then integrating by parts we get for $\varepsilon>0$ small enough,

$$
\begin{aligned}
\int_{\varepsilon}^{T} \int_{0}^{2} \bar{u}\left(\frac{\partial}{\partial t}+\frac{\partial^{2}}{\partial x^{2}}\right)\left(\psi_{\alpha} t v\right) t^{\alpha} d z \\
=\left.\int_{\varepsilon}^{T}\left[\bar{u} \frac{\partial v}{\partial x}-v \frac{\partial \bar{u}}{\partial x}\right]\right|_{x=0} ^{x=2} \psi_{\alpha} t^{\alpha+1} d t \\
\quad+\left.\int_{0}^{2} \psi_{\alpha} t^{\alpha+1} \bar{u} v\right|_{t=\varepsilon} ^{t=T} d x-\int_{\varepsilon}^{T} \int_{0}^{2} \alpha \psi_{\alpha} \bar{u} v t^{\alpha} d z \\
=-\int_{0}^{2} \frac{\varepsilon^{\alpha+1}}{\alpha+1} \overline{u(x, \varepsilon)} v(x, \varepsilon) d x-\int_{\varepsilon}^{T} \int_{0}^{2} \alpha \psi_{\alpha} \bar{u} v t^{\alpha} d z
\end{aligned}
$$

Lemma 6 and the dominated convergence theorem imply $(g, u)_{L_{\alpha}^{2}\left(\Omega_{T}\right)}=$ 0 .

Corollary 11. The mapping $P_{\alpha}: L^{\infty}\left(\Omega_{T}\right) \rightarrow \mathcal{B}_{T}$ is onto.

From Proposition 10 we have the following

Corollary 12. If $v \in \mathcal{B}_{T}$ then $\sup \left\{t^{1 / 2}\left|\frac{\partial v}{\partial x}(x, t)\right|:(x, t) \in \Omega_{T}\right\}<\infty$. 
Proposition 13. For $\alpha>-1$, the bilinear form $\langle\cdot, \cdot\rangle_{\alpha}: b_{\alpha}^{1}\left(S_{T}\right) \times \mathcal{B}_{T} \rightarrow$ $\mathbb{C}$ given by

$$
\langle u, v\rangle_{\alpha}=\int_{\Omega_{T}} u(z) \overline{\left(\mathcal{L}_{\alpha}^{1} v\right)(z)} t^{\alpha} d z
$$

is continuous, and $\langle u, v\rangle_{\alpha}=(u, v)_{b_{\alpha}^{2}\left(S_{T}\right)}$ for $u \in b_{\alpha}^{2}\left(S_{T}\right), v \in \mathcal{B}_{T}$.

Proof. For $u \in b_{\alpha}^{1}\left(S_{T}\right), v \in \mathcal{B}_{T}$, we have

$$
\left|\langle u, v\rangle_{\alpha}\right| \leq\|u\|_{b_{\alpha}^{1}\left(S_{T}\right)}\left\|\mathcal{L}_{\alpha}^{1} v\right\|_{L^{\infty}\left(\Omega_{T}\right)} \leq C\|u\|_{b_{\alpha}^{1}\left(S_{T}\right)}\|v\|_{\mathcal{B}_{T}} .
$$

On the other hand, if $u \in b_{\alpha}^{2}\left(S_{T}\right), v \in \mathcal{B}_{T}$

$$
\begin{aligned}
\langle u, v\rangle_{\alpha} & =\left(u, \mathcal{L}_{\alpha}^{1} v\right)_{b_{\alpha}^{2}\left(S_{T}\right)} \\
& =\left(P_{\alpha} u, \mathcal{L}_{\alpha}^{1} v\right)_{b_{\alpha}^{2}\left(S_{T}\right)} \\
& =\left(u, P_{\alpha} \mathcal{L}_{\alpha}^{1} v\right)_{b_{\alpha}^{2}\left(S_{T}\right)} \\
& =(u, v)_{b_{\alpha}^{2}\left(S_{T}\right)} .
\end{aligned}
$$

Proof of Theorem 2. Consider the linear mapping $\Phi_{\alpha}: \mathcal{B}_{T} \rightarrow\left(b_{\alpha}^{1}\left(S_{T}\right)\right)^{*}$ given by $\Phi_{\alpha} v=\langle\cdot, v\rangle_{\alpha}$. By the previous proposition $\Phi_{\alpha}$ is continuous, and injective: If $\Phi_{\alpha} v=0$ then $\langle v, v\rangle_{\alpha}=(v, v)_{b_{\alpha}^{2}\left(S_{T}\right)}=0$, therefore $v \equiv 0$.

Let $F \in\left(b_{\alpha}^{1}\left(S_{T}\right)\right)^{*}$, by the Hahn-Banach theorem the operator $F$ can be extended continuously to $L^{1}\left(\Omega_{T}, t^{\alpha} d z\right)$, and the Riesz representation theorem implies the existence of $\varphi \in L^{\infty}\left(\Omega_{T}\right)$ such that

$$
F u=\int_{\Omega_{T}} u(z) \overline{\varphi(z)} t^{\alpha} d z, \text { for all } u \in b_{\alpha}^{1}\left(S_{T}\right) .
$$

In particular, for $u \in b_{\alpha}^{2}\left(S_{T}\right)$ we have

$$
\begin{aligned}
F u & =(u, \varphi)_{L_{\alpha}^{2}\left(\Omega_{T}\right)}=\left(P_{\alpha} u, \varphi\right)_{L_{\alpha}^{2}\left(\Omega_{T}\right)} \\
& =\left(u, P_{\alpha} \varphi\right)_{b_{\alpha}^{2}\left(S_{T}\right)}=\left\langle u, P_{\alpha} \varphi\right\rangle_{\alpha},
\end{aligned}
$$

where the last equality follows from the previous proposition. Since $b_{\alpha}^{2}\left(S_{T}\right)$ is dense in $b_{\alpha}^{1}\left(S_{T}\right)$, then $F=\Phi_{\alpha}\left(P_{\alpha} \varphi\right)$. By the open mapping theorem we have that $\Phi_{\alpha}$ is an isomorphism.

We finish with a simple example of Bloch-type temperature functions,

Example 14. Suppose $\left(a_{n}\right)_{n}$ is a bounded sequence of complex numbers and suppose $\left(\lambda_{n}\right)_{n}$ is a sequence of positive integers with $\lambda_{n} / \lambda_{n-1} \geq \sqrt{\lambda}$, where $1<\lambda<\infty$. Let

$$
v(x, t)=\sum_{n=1}^{\infty} a_{n} e^{-\pi^{2} \lambda_{n}^{2} t+\pi \lambda_{n} i x} .
$$

Since $e^{-x} \leq C_{\sigma} x^{-\sigma}$ for $x>0, \sigma \geq 0$ then $v \in H\left(S_{T}\right)$, and

$$
\left|\frac{\partial v}{\partial t}(x, t)\right| \leq M \sum_{n=1}^{\infty} \lambda_{n}^{2}\left(e^{-\pi^{2} t}\right)^{\lambda_{n}^{2}},
$$


where $M>0$ is a constant.

Setting $z=e^{-\pi^{2} t}$, and $K=\lambda /(\lambda-1)$ we have that $0<z<1,1<K<\infty$.

Then,

$$
\lambda_{1}^{2}|z|^{\lambda_{1}^{2}} \leq|z|+\cdots+|z|^{\lambda_{1}^{2}} \leq K\left(|z|+\cdots+|z|^{\lambda_{1}^{2}}\right) .
$$

Since $\lambda_{n}^{2} \leq K\left(\lambda_{n}^{2}-\lambda_{n-1}^{2}\right)$ for every $n \geq 2$, we have

$$
\lambda_{n}^{2}|z|^{\lambda_{n}^{2}} \leq K\left(|z|^{\lambda_{n-1}^{2}+1}+\cdots+|z|^{\lambda_{n}^{2}}\right) .
$$

Let $\delta>0$ small enough and $C_{\delta}>0$ a constant such that $t \leq C_{\delta}\left(1-e^{-\pi^{2} t}\right)$ for $0<t<\delta$, therefore

$$
\left|\frac{\partial v}{\partial t}(x, t)\right| \leq M K \sum_{n=1}^{\infty}|z|^{n} \leq \frac{M K}{1-e^{-\pi^{2} t}} \leq \frac{C_{\delta} M K}{t} .
$$

Since $\frac{\partial v}{\partial t} \in C(\mathbb{R} \times[\delta, \infty))$ we obtain that $v \in \mathcal{B}_{T}$.

Acknowledgement. This article was written while visiting the Universitat de València, Spain, on posdoctoral stay; the author has been supported by Programa de Apoyos para la Superación del Personal Académico de la DGAPA of the Universidad Nacional Autónoma de México. The author thanks the Departament de Anàlisi Matemàtica of the Universitat de València for its hospitality and support.

\section{References}

[1] S. Bell, A duality theorem for harmonic functions, Michigan Math. J., 29 (1) (1982), 123-128.

[2] J.R. Cannon, The One-Dimensional Heat Equation, Encyclopedia of Mathematics and its Applications, 23, Addison-Wesley Publishing Company, Advanced Book Program, Reading, MA, 1984.

[3] B. Coupet, Le dual des functions holomorphes intégrables sur un domaine strictement pseudo-convexe, In : Proceedings of the American Mathematical Society, 102 (3), (1988), 493-501.

[4] H. Hedenmalm, B. Korenblaum and K. Zhu, Theory of Bergman Spaces, Graduate Texts in Mathematics, Vol. 199, Springer Verlag, New York, 2000.

[5] E. Ligocka, On the reproducing kernel for harmonic functions and the space of Bloch harmonic functions on the unit ball in $R^{n}$, Studia Math., 87 (1) (1987), 23-32.

[6] J. Motos and S. Pérez-Esteva, On Bell's duality theorem for harmonic functions, Studia Math., 137 (1) (1999), 49-60. 
[7] M. López-García, Bergman spaces of temperature functions on a cylinder, Int. J. Math. Math. Sci., 2003 (19) (2003), 1193-1213.

[8] M. López-García, An atomic decomposition for the Bergman space of temperature functions on a cylinder, Bol. Soc. Mat. Mexicana, 11 (3) (2005), 101-119.

[9] D.V. Widder, The Heat Equation, Pure and Applied Mathematics, Vol. 67, Academic Press, New York, 1975.

Instituto de Matemáticas

Universidad Nacional Autonóma de México

Circuito Exterior Ciudad Universitaria

México D.F., C.P. 04510

México

(E-mail : flopez@matem.unam.mx) 


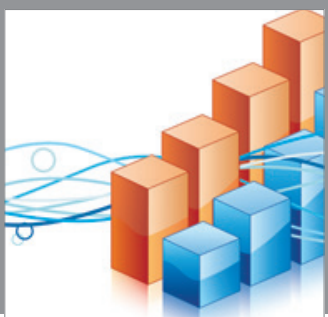

Advances in

Operations Research

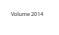

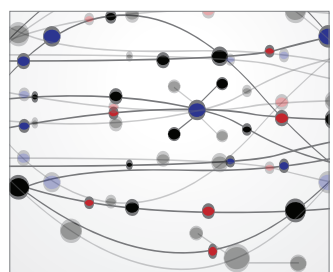

\section{The Scientific} World Journal
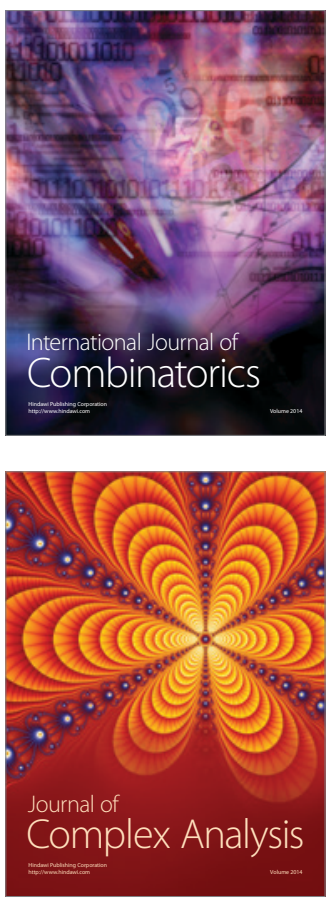

International Journal of

Mathematics and

Mathematical

Sciences
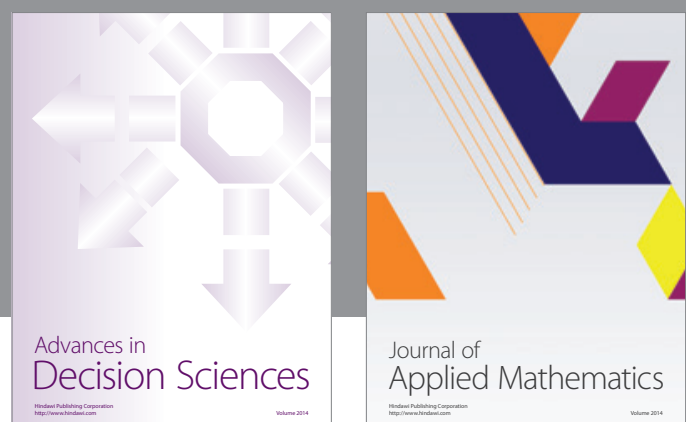

Journal of

Applied Mathematics
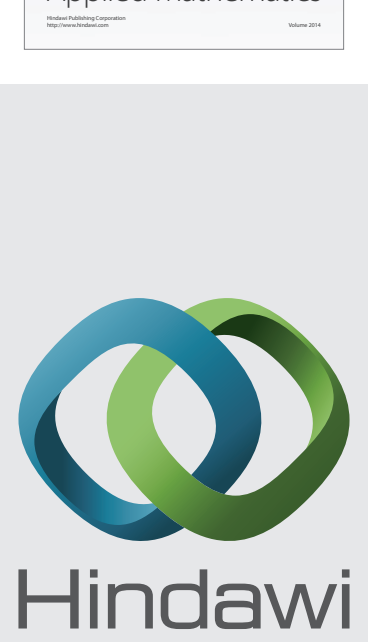

Submit your manuscripts at http://www.hindawi.com
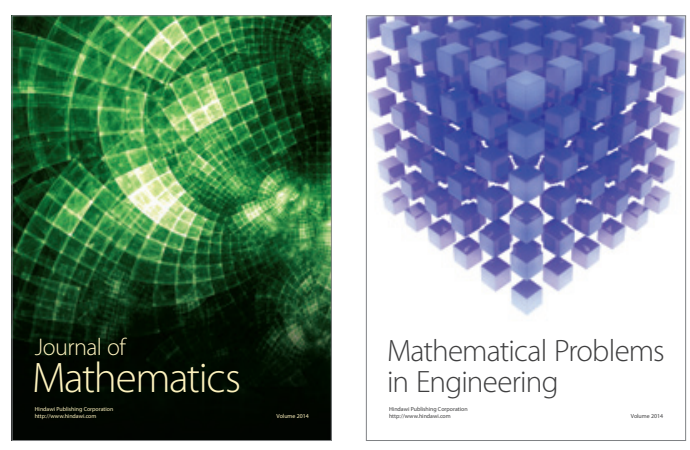

Mathematical Problems in Engineering
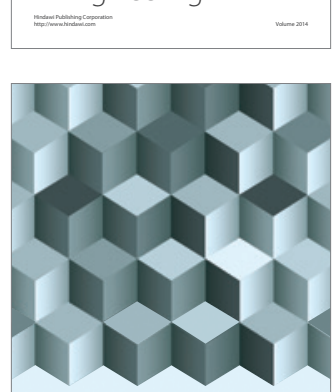

Journal of

Function Spaces
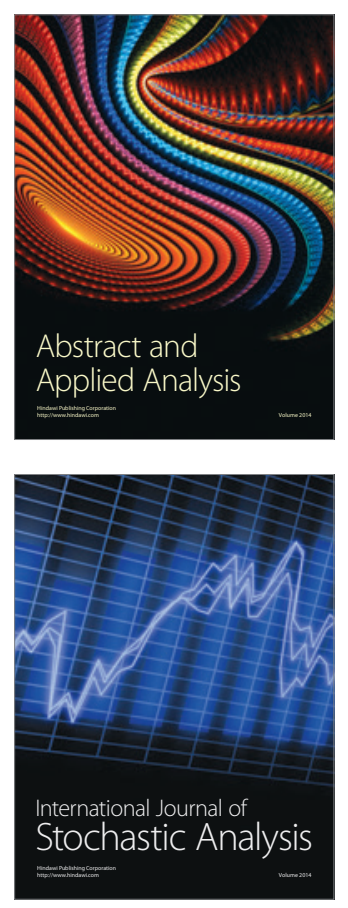

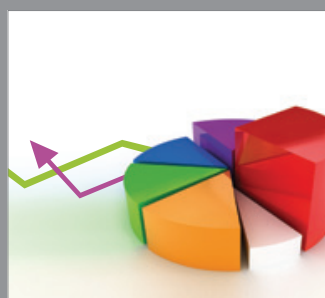

ournal of

Probability and Statistics

Promensencen
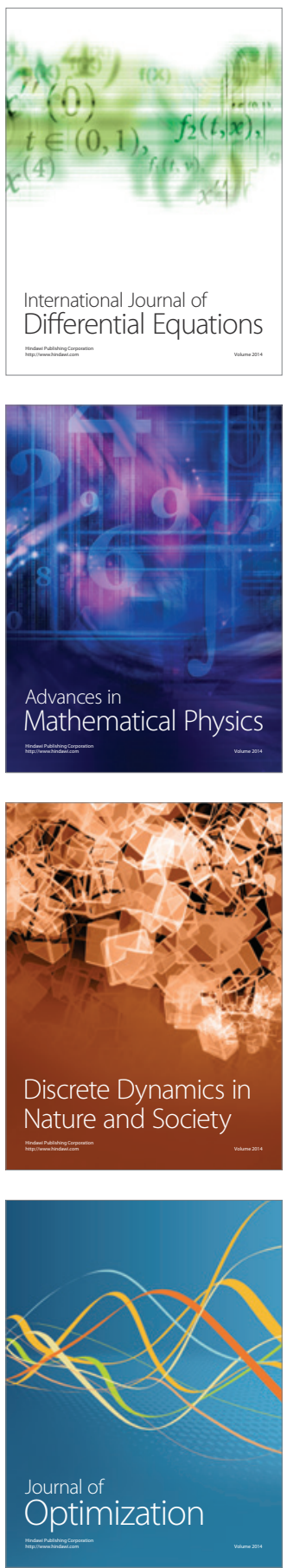